\title{
Range of the Safe Retaining Pressures of a Pressurized Tunnel Face by a Probabilistic Approach
}

\author{
Guilhem Mollon; Daniel Dias²; and Abdul-Hamid Soubra, M.ASCE ${ }^{3}$
}

\begin{abstract}
A probabilistic analysis of the face stability of a pressurized tunnel is undertaken in this article. First, two existing velocity fields based on the limit analysis theory are briefly described. They allow one to compute the values of the critical pressures of collapse and blowout of a pressurized tunnel face in cases of both frictional and nonfrictional soils. These models, which have the great advantage of a low computational cost, are validated by comparison with a computationally expensive numerical model. Then, an efficient probabilistic method called collocation-based stochastic response surface methodology (CSRSM) is applied on these velocity fields to perform the uncertainty propagation. This method makes it possible to compute the probability of failure of the tunnel face against both collapse and blowout. In the case of a frictional soil, it appears that the blowout of the face is extremely unlikely and that the collapse is the only probable failure mode. On the contrary, in a purely cohesive soil, it appears that both failure modes are likely to appear and should be considered in the analysis. Finally, this paper presents a discussion concerning the application of the proposed probabilistic method for an economic and safe design of a pressurized shield. DOI: 10.1061/(ASCE)GT.1943-5606.0000911. (C) 2013 American Society of Civil Engineers.
\end{abstract}

CE Database subject headings: Tunnels; Pressure; Probability; Design.

Author keywords: Tunnel face stability; Collapse; Blowout; CSRSM; Uncertainty propagation; Probabilistic design.

\section{Introduction}

In tunneling projects, the stability of the tunnel face is a major issue. When the excavation is performed using a pressurized shield, this stability is ensured by applying a fluid pressure, called $\sigma_{t}$, to the tunnel face. The fluid may be compressed air, slurry, or the earth excavated by the tunnel. In this paper, a uniform pressure is considered, which may be associated with compressed air stabilization. Instability of the face may occur if the fluid pressure is too low [in this case, the failure mode is called collapse, and the motion of the soil is directed toward the face, as shown in Fig. 1(a)] or if it is too high [in this case, the failure mode is called blowout, and the motion of the soil is directed toward the ground surface, as shown in Fig. 1(b)]. This means that the range of the safe fluid pressures that can be applied to a tunnel face is bounded by a lower value (the critical collapse pressure, called $\sigma_{c}$ ) and an upper value (the critical blowout pressure, called $\sigma_{b}$ ). For the sake of simplicity, it is supposed in this article that there is no surcharge loading $\sigma_{s}$ on the ground surface.

While the models of prediction of the tunnel face instability become more and more accurate, an important issue for the designers is to deal with the uncertainties related to the input parameters of such models. In engineering practice, this uncertainty is dealt with using

\footnotetext{
${ }^{1}$ Research Associate, Dept. of Civil and Environmental Engineering, Hong Kong Univ. of Science and Technology, Clear Water Bay, Kowloon, Hong Kong (corresponding author). E-mail: guilhem.mollon@gmail.com

${ }^{2}$ Professor, Laboratoire d'Etude des Transferts en Hydrologie et Environnement, Univ. Joseph Fourier Grenoble 1, 38041 Saint Martin d'Hères, France. Email: d.dias69@gmail.com

${ }^{3}$ Professor, Dept. of Civil Engineering, Univ. of Nantes, 44600 Saint Nazaire, France. E-mail: abed.soubra@univ-nantes.fr

Note. This manuscript was submitted on September 15, 2012; approved on February 4, 2013; published online on February 6, 2013. Discussion period open until April 1, 2014; separate discussions must be submitted for individual papers. This paper is part of the Journal of Geotechnical and Geoenvironmental Engineering, Vol. 139, No. 11, November 1, 2013. (c)ASCE, ISSN 1090-0241/2013/11-1954-1967/\$25.00.
}

a global safety factor. The target safety factor adopted in design is based on engineering judgment and/or design codes. More recent and appealing approaches for a safe design of civil engineering projects in the presence of uncertainties are based on probabilistic methods. Such methods require assessing the propagation of the uncertainties from the input variables to the system output that is used in design. This propagation can be performed by a number of methods, and the main issue is related to the very high computational cost of most of these methods.

Several attempts have been made by the authors of the present paper to perform a probabilistic study of the collapse of a pressurized tunnel face, using simplified deterministic models and/or approximate probabilistic methods such as the first-order reliability method (FORM) or the response surface method (RSM), as described in Mollon et al. (2009a, b). The use of simplified deterministic models and approximate probabilistic methods was related to the high computational costs of the numerical deterministic models and the Monte Carlo probabilistic approach (which are believed to lead to accurate solutions). However, efforts have been made since then by the authors of this paper (1) to build up new deterministic analytical models providing accurate critical pressures with a limited time cost (Mollon et al. 2011b, 2012); and (2) to implement an efficient probabilistic method called collocation-based stochastic response surface methodology (CSRSM), which provides accurate probabilistic results from a limited number of calls of the deterministic models (Mollon et al. 2011a). Hence, this article aims at applying CSRSM to these new deterministic models. Because both the deterministic models and the probabilistic approach have been improved, this study is expected to provide more accurate probabilistic results than the previously published ones with a smaller computation time. Moreover, this paper focuses not only on the face collapse but also on the blowout phenomenon of this face, allowing one to obtain the value of the failure probability, not only against collapse, but against both modes of failure (collapse and blowout). Finally, this paper presents a discussion concerning the application of the proposed probabilistic method for an economic and safe design of a pressurized shield. 


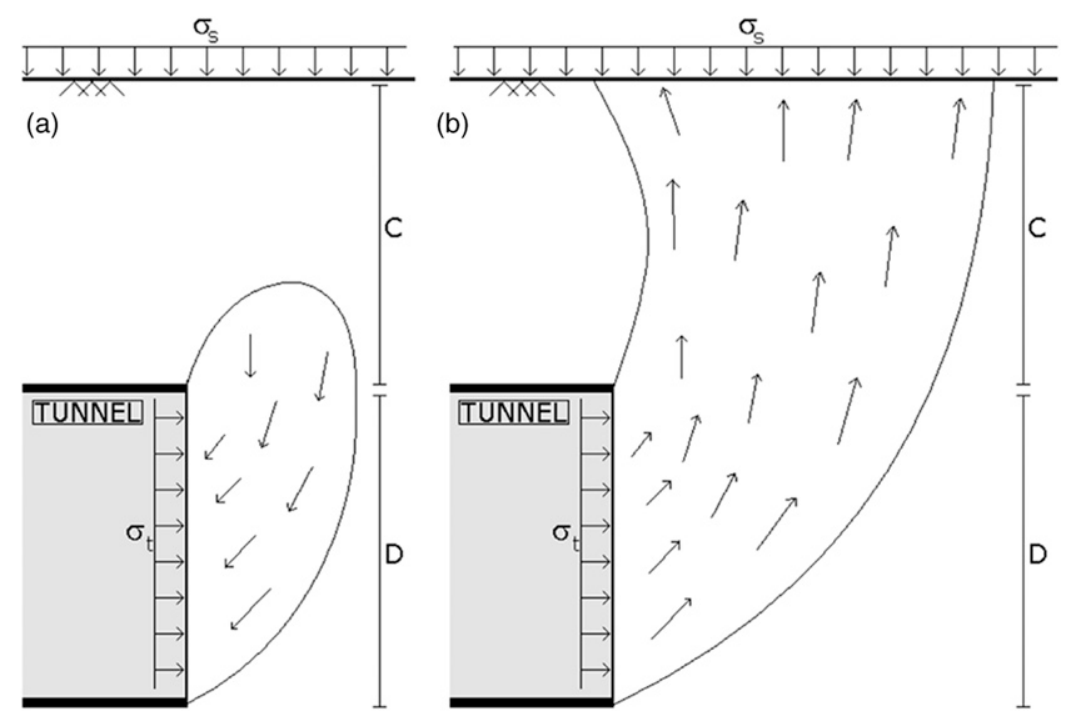

Fig. 1. Layout of the two modes of face failure: (a) collapse; (b) blowout

\section{Overview of Existing Deterministic Models}

The face stability of circular tunnels driven by pressurized shields has been investigated by several authors in the literature. For the case of a frictional soil, some authors performed experimental tests (Chambon and Corté 1994; Takano et al. 2006). Others (Leca and Dormieux 1990; Anagnostou and Kovari 1996; Mollon et al. 2009a, b, 2010, 2011b) performed analytical or numerical approaches. The model by Leca and Dormieux (1990) is based on the kinematic theorem of limit analysis. This model was successively improved using several kinematically admissible velocity fields (see Mollon et al. 2009a, 2010, 2011b). The collapse and blowout velocity fields by Mollon et al. (2011b) are the most critical ones and will be referred to in this paper as the M1 model. This model is briefly presented in this paper. Concerning the case of a purely cohesive soil, one may cite the investigations by Broms and Bennermark (1967), Davis et al. (1980), Ellstein (1986), Augarde et al. (2003), Osman et al. (2006), and Klar et al. (2007), among others. More recently, Mollon et al. (2012) have presented a velocity field in the framework of the kinematic theorem of limit analysis. This velocity field (referred to in this paper as the M2 model) is an extension of the work by Osman et al. (2006) and Klar et al. (2007). It will also be briefly presented in this paper.

The following subsections aim at briefly presenting (1) a computationally expensive numerical model called the numerical model of reference, which is believed to give somewhat accurate solutions; (2) the analytical model M1 by Mollon et al. (2011b) for frictional soils; (3) the analytical model M2 by Mollon et al. (2012) for purely cohesive soils; and last (4) a validation of the M1 and M2 models (via a comparison of their results with those given by the numerical model of reference) for use in a probabilistic framework.

\section{Numerical Model of Reference}

The numerical model is based on numerical simulations using FLAC $3 D$ software. It is shown in Fig. 2. The model dimensions are 50 $\times 40 \times 26 \mathrm{~m}$ in the transversal, longitudinal, and vertical directions, respectively, for a tunnel diameter $D=10 \mathrm{~m}$ and a cover-to-depth ratio $C / D=1$. The vertical dimension may be modified to deal with different $C / D$ ratios. The soil is assigned an elastic perfectly plastic constitutive model based on Mohr-Coulomb failure criterion. An

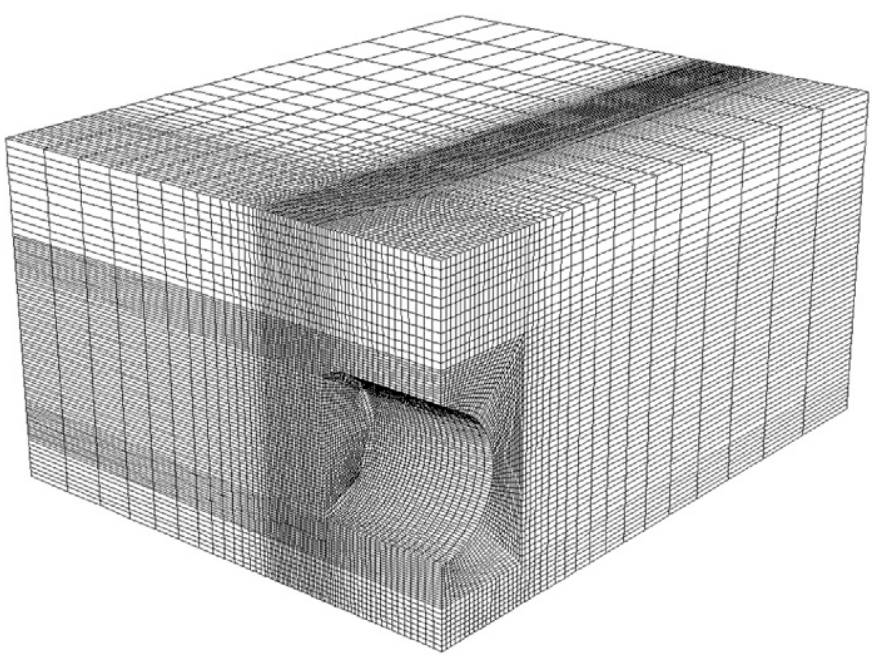

Fig. 2. Numerical model introduced in FLAC $3 D$

associated flow rule was used to make the comparison meaningful, but it should be noted that the influence of the dilation angle on the critical collapse and blowout pressure is rather limited (Mollon 2010). The use of the present numerical model in a probabilistic framework is very inconvenient because its computation time is close to $50 \mathrm{~h}$. Indeed, an accurate estimate of $\sigma_{c}$ or $\sigma_{b}$ requires a very fine mesh $(215,000$ elements). Details on this issue are provided in Mollon et al. (2011c) in a two-dimensional (2D) framework. Notice however that this numerical model may be used as a reference tool to validate other less costly deterministic models. A purely frictional soil $\left(\varphi=30^{\circ}\right.$, $c=0 \mathrm{kPa})$ and a purely cohesive soil $\left(\varphi=0^{\circ}, c=30 \mathrm{kPa}\right)$ were introduced in the numerical model, and the values of $\sigma_{c}$ were computed for both cases, giving, respectively $\sigma_{c}=23.7 \mathrm{kPa}$ and $\sigma_{c}=53.1 \mathrm{kPa}$. Slightly lower pressures than $\sigma_{c}$ were applied to both soils to trigger instability, and the deformations of the mesh in the plane of symmetry of the tunnel are shown in Fig. 3. This figure clearly shows that the collapse phenomenon is very different in frictional and nonfrictional soils. Indeed, the face collapse in a frictional soil involves a rotational motion of a single rigid block. Such a failure exhibits a thin shear band located between the moving block and the soil at rest. On the other 


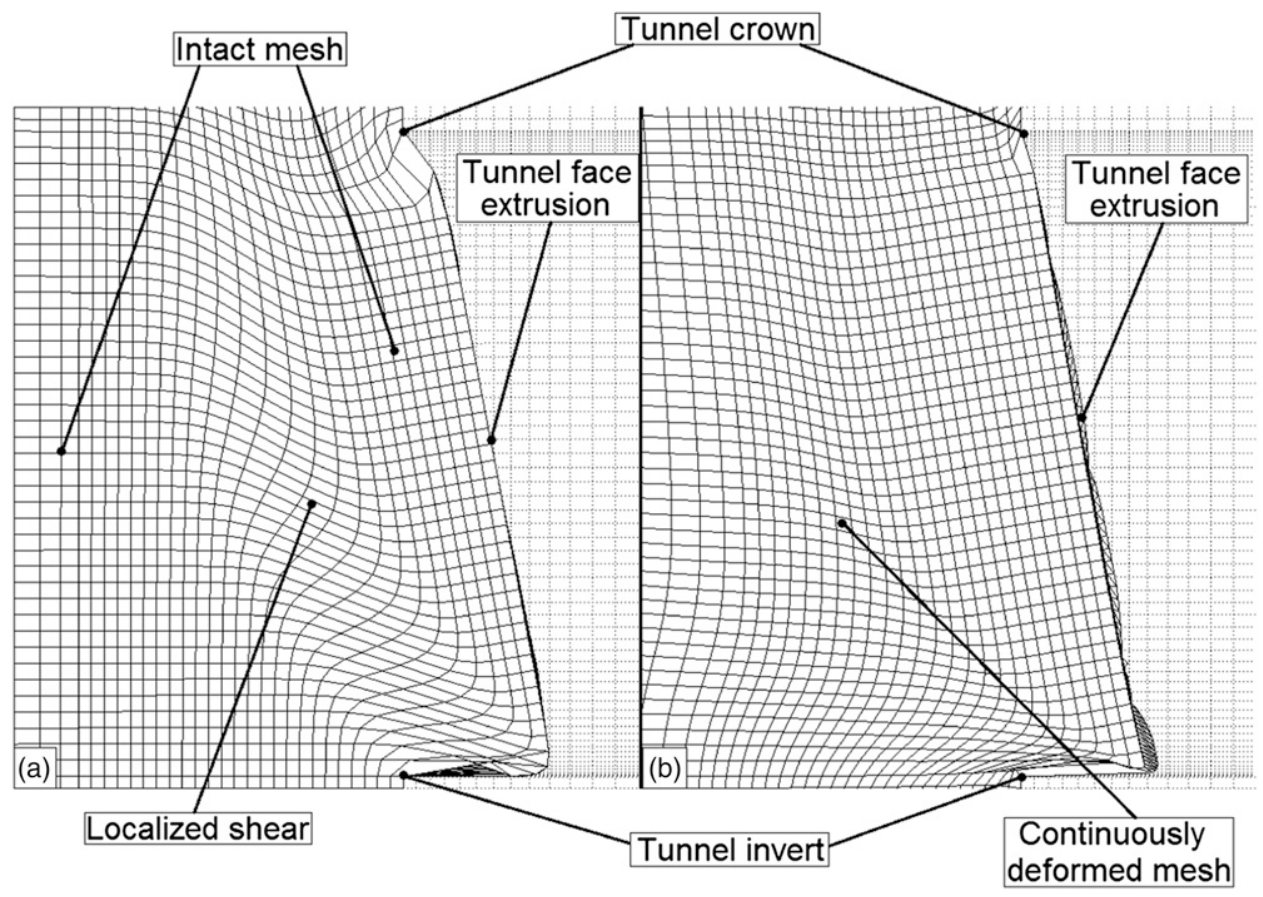

Fig. 3. Deformation of the numerical mesh during a face collapse: (a) $\varphi=30^{\circ}$ and $c=0 \mathrm{kPa}$; (b) $\varphi=0^{\circ}$ and $c=30 \mathrm{kPa}$

hand, in a purely cohesive soil, one may observe a continuous deformation of the soil mass without any shear band. Based on these observations, it seems that a single analytical model would not be able to provide accurate estimates of the critical pressures for both frictional and nonfrictional soils. Two different limit analysis models based on the kinematic approach are used herein for frictional and nonfrictional soils. These models are described in the next subsections. Notice that because of the use of the kinematic theorem of limit analysis, one obtains a rigorous lower bound of $\sigma_{c}$ and a rigorous upper bound of $\sigma_{b}$. The M1 and M2 models therefore provide a rigorous bracketing of the admissible interval for the applied face pressure $\sigma_{t}$ for both frictional and nonfrictional soils.

\section{Overview of M1 Analytical Model for Frictional Soils}

The deterministic model used for the computation of $\sigma_{c}$ and $\sigma_{b}$ in the case of a frictional soil (with or without cohesion) will be called M1 in this article. It was extensively presented in Mollon et al. (2011b), and is briefly described hereafter. Two rotational rigid block mechanisms are considered for the active (collapse) and passive (blowout) cases. Fig. 4 shows views of the collapse and blowout mechanisms for $\varphi=30^{\circ}$ and $c=0 \mathrm{kPa}$. A cylindrical rotational velocity field is considered for the two mechanisms. This means that both mechanisms rotate with a uniform angular velocity $\omega$ around a horizontal axis. The only difference between the collapse and blowout cases is the direction of the rotational motion. The failure mechanisms are entirely defined by the position of the axis of rotation, and therefore depend on only two parameters. This is because the envelope of the moving block is constructed point by point in such a manner to respect the normality condition imposed by limit analysis at the different points along the velocity discontinuity surface, and such that its intersection with the tunnel face corresponds to the circular contour of this face. Notice that in the blowout case, the failure mechanism outcrops at the ground surface [Fig. 4(b)]. In the collapse case, it may be the same, but the most usual case corresponds to a mechanism which closes to itself without reaching the ground surface [Fig. 4(a)]. For both mechanisms, the computation time is nearly equal to 3 min using the four processors of a Intel Core 2 Quad CPU $2.4 \mathrm{GHz}$ computer (Santa Clara, California).

\section{Overview of M2 Analytical Model for Purely Cohesive Soils}

The numerical results of Fig. 3 have shown that failure mechanisms involving the motion of rigid blocks are not relevant for the failure of a tunnel face in a purely cohesive soil. To explore suitable velocity fields, one has to consider failure patterns involving continuous deformation of the soil mass between the tunnel face and the ground surface with no velocity jump at the envelope of the velocity field. This is the aim of the M2 analytical model. This model was developed in Mollon et al. (2012) and is briefly described hereafter in the collapse case; the blowout case being straightforward. As shown in Fig. 5, the external envelope of the velocity field is defined by a collection of circles. The envelope of the velocity field appears similar to a torus of center $\mathrm{O}$, but it should be noted that this is not an actual torus [more details are provided in Mollon et al. (2012)]. The soil velocity during failure is zero on this envelope, and follows an asymmetric parabolic profile inside it.

The normality condition in a purely cohesive soil implies that any plastic deformation may occur without any change of volume. This assumption is used herein to compute the components of the velocity field everywhere in the somewhat-toric domain. The computation of a critical collapse or blowout pressure by the M2 model takes approximately $20 \mathrm{~s}$, using the four processors of a $2.4 \mathrm{GHz}$ quad-core CPU computer.

\section{Numerical Validation of M1 and M2 Analytical Models}

The numerical model introduced in FLAC3D and shown in Fig. 2 is used to evaluate the accuracy of the M1 and M2 analytical models. As stated earlier in this article, the M1 and M2 models developed in 
(a)

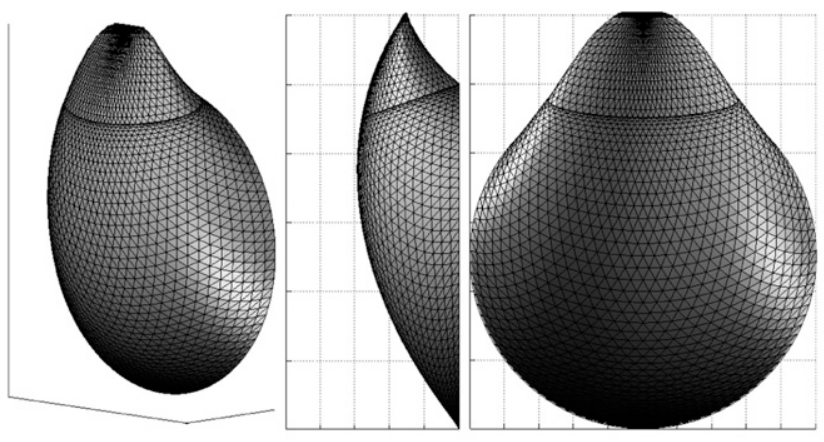

(b)

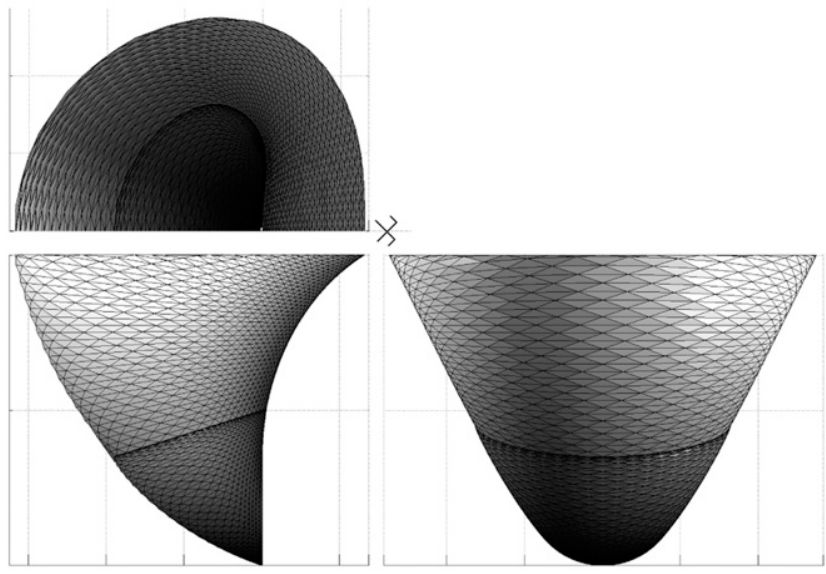

Fig. 4. Layout of the face failure as predicted by the M1 model for the case $\varphi=30^{\circ}, c=0 \mathrm{kPa}$, and $C / D=1$ : (a) collapse; (b) blowout

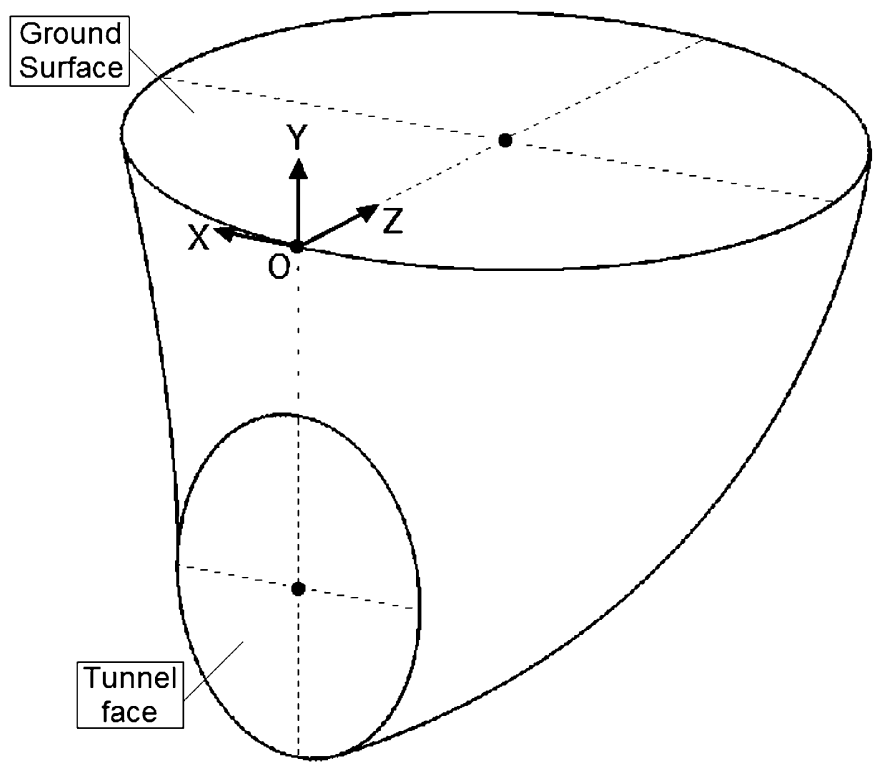

Fig. 5. Layout of the 3D envelope of the M2 model in case of collapse or blowout

the framework of the kinematic theorem of the limit analysis theory provide rigorous lower bounds of $\sigma_{c}$ and rigorous upper bounds of $\sigma_{b}$. To be accurate, a limit analysis model should provide a value of $\sigma_{c}$ (respectively, $\sigma_{b}$ ) as high (respectively, as low) as possible. Tables 1 and 2 provide a comparison between the values of $\sigma_{c}$ and $\sigma_{b}$ provided by the present kinematical models and those given by the
Table 1. Critical Collapse and Blowout Pressures as Obtained by the M1 Analytical Model and the FLAC3D Numerical Model for $D=10 \mathrm{~m}$ and $\gamma=18 \mathrm{kN} / \mathrm{m}^{3}$

\begin{tabular}{|c|c|c|c|c|c|c|c|c|}
\hline \multirow[b]{3}{*}{$C / D$} & \multicolumn{4}{|c|}{$\varphi=17^{\circ} ; c=7 \mathrm{kPa}$} & \multicolumn{4}{|c|}{$\varphi=25^{\circ} ; c=10 \mathrm{kPa}$} \\
\hline & \multicolumn{2}{|c|}{$\begin{array}{c}\text { Collapse } \\
\text { pressure }(\mathrm{kPa})\end{array}$} & \multicolumn{2}{|c|}{$\begin{array}{c}\text { Blowout pressure } \\
(\mathrm{kPa})\end{array}$} & \multicolumn{2}{|c|}{$\begin{array}{c}\text { Collapse } \\
\text { pressure }(\mathrm{kPa}) \\
\end{array}$} & \multicolumn{2}{|c|}{$\begin{array}{c}\text { Blowout pressure } \\
(\mathrm{kPa})\end{array}$} \\
\hline & M1 & $F L A C 3 D$ & M1 & $F L A C 3 D$ & M1 & $F L A C 3 D$ & M1 & $F L A C 3 D$ \\
\hline 0.6 & 37.8 & 36.8 & 682.4 & 635 & 13.9 & 11.7 & $1,112.2$ & 1,091 \\
\hline 0.8 & 37.8 & 37.2 & 878.6 & 864 & 13.9 & 12.1 & $1,487.3$ & 1,521 \\
\hline 1 & 37.8 & 37.3 & $1,096.6$ & 1,113 & 13.9 & 12.2 & $1,903.5$ & 2,004 \\
\hline 1.5 & 37.8 & 37.3 & $1,777.1$ & 1,842 & 13.9 & 12.2 & $3,301.2$ & 3,488 \\
\hline 2 & 37.8 & 37.4 & $2,637.7$ & 2,740 & 13.9 & 12.2 & $5,213.3$ & 5,337 \\
\hline & 37.8 & 37.4 & $5,243.1$ & 5,253 & 13.9 & 12.2 & - & 一 \\
\hline
\end{tabular}

Table 2. Critical Collapse and Blowout Pressures as Obtained by the M2 Analytical Model and by the FLAC3D Numerical Model for $D=10 \mathrm{~m}$ and $\gamma=18 \mathrm{kN} / \mathrm{m}^{3}$

\begin{tabular}{|c|c|c|c|c|c|c|c|c|}
\hline \multirow[b]{3}{*}{$C / D$} & \multicolumn{4}{|c|}{$c_{u}=20 \mathrm{kPa}$} & \multicolumn{4}{|c|}{$c_{u}=30 \mathrm{kPa}$} \\
\hline & \multicolumn{2}{|c|}{$\begin{array}{c}\text { Collapse } \\
\text { pressure }(\mathrm{kPa}) \\
\end{array}$} & \multicolumn{2}{|c|}{$\begin{array}{c}\text { Blowout } \\
\text { pressure }(\mathrm{kPa})\end{array}$} & \multicolumn{2}{|c|}{$\begin{array}{c}\text { Collapse } \\
\text { pressure }(\mathrm{kPa}) \\
\end{array}$} & \multicolumn{2}{|c|}{$\begin{array}{c}\text { Blowout pressure } \\
(\mathrm{kPa})\end{array}$} \\
\hline & M2 & $F L A C 3 D$ & M2 & $F L A C 3 D$ & M2 & $F L A C 3 D$ & M2 & $F L A C 3 L$ \\
\hline 6 & 84.8 & 86.7 & 316.0 & 207 & 20.2 & 22.1 & 386.2 & 358 \\
\hline 0.8 & 106.5 & 108.8 & 388.5 & 342 & 34.6 & 37.5 & 473.3 & 419 \\
\hline 1 & 129.4 & 132.9 & 443.9 & 392 & 50.7 & 53.1 & 538.2 & 474 \\
\hline 1.5 & 192.1 & 199.1 & 565.0 & 509 & 97.8 & 106.3 & 675.9 & 604 \\
\hline 2 & 256.9 & 272.7 & 683.2 & 617 & 150.5 & 169.5 & 808.5 & 726 \\
\hline 3 & 398.9 & 447.9 & 906.0 & 814 & 272.1 & 340.7 & $1,054.0$ & 922 \\
\hline
\end{tabular}

numerical FLAC $3 D$ model. In the case of frictional soils, Table 1 shows that the M1 model provides solutions that are not far from the values provided by the numerical model. Similar agreement was obtained for several cases of frictional soils with or without cohesion (not shown in this paper). Furthermore, as was mentioned in Mollon et al. (2011b), the present velocity fields provide the best bounds of the critical pressures (that is, the highest values of $\sigma_{c}$ and the lowest values of $\sigma_{b}$ ). The same conclusions found for the M1 model can be drawn for the M2 model. The M1 and M2 models can therefore be applied with confidence in a probabilistic context instead of the numerical model, with a limited loss of accuracy but for a much lower computational cost.

\section{Probabilistic Study}

This section aims at investigating the effect of the uncertainties related to the soil properties and the applied face pressure on the tunnel face stability. Six soil configurations are considered: (1) two purely frictional soils corresponding to sands (called S1 and S2); (2) two frictional and cohesive soils corresponding to drained clays (called DC1 and DC2); and (3) two purely cohesive soils corresponding to undrained clays (called UC1 and UC2). The mean values of the soil shear strength parameters, the soil unit weight, the tunnel diameter and the tunnel cover depth corresponding to each configuration are summarized in Table 3 . This table provides the deterministic values of $\sigma_{c}$ and $\sigma_{b}$ for the six configurations as provided by the models M1 (for S1, S2, DC1, and DC2) and M2 (for UC1 and UC2).

In the following, one first presents the uncertain input parameters used in the analysis. This is followed by the method used for the propagation of the uncertainties from the input data to the system responses $\left(\sigma_{c}\right.$ and $\left.\sigma_{b}\right)$. Then, the failure probability against both 
collapse and blowout of the tunnel face is computed. Finally, a design based on a target failure probability is proposed.

\section{Uncertain Input Data}

Five uncertain parameters (see Table 4) were considered in the analysis. Three probabilistic scenarios were studied because the variability of the input random variables is generally not known with accuracy. The neutral scenario makes use of some classical values of the coefficients of variation (COVs) of the random variables, while the optimistic and pessimistic scenarios deal with smaller and larger values of the COVs, respectively. All the values of the COVs are provided in Table 4. It should be mentioned that the variability of the soil shear strength parameters $(\varphi, c)$ is quite well documented (Phoon and Kulhawy 1999). This is related to the natural variability of the soil and to the possible measurement errors. The unit weight is a soil characteristic that is easy to assess, which explains the low values of COV for this variable. In the same way, the cover depth $C$ is controlled by the operator of the tunnel boring machine (TBM), and is subject to a low level of uncertainty. The COV of the fluid pressure $\sigma_{t}$ applied at the tunnel face is very low (5\%) in the optimistic scenario, and quite high $(25 \%)$ in the pessimistic one, depending on the level of control of this pressure (related to the tunnel machinery and human calibration). This variability also accounts for the uncertainty on the soil behind this pressure, especially its permeability to the retaining fluid.

In the present paper, each input random variable will be modeled using two different probability density functions (PDFs). In the socalled normal variables case, the five variables are considered as Gaussian, while in the so-called nonnormal variables case, the soil friction angle $\varphi$ is assumed to follow a beta distribution (bounded by 0 and $45^{\circ}$ ) and the four other variables are assumed to follow

Table 3. Soil and Geometrical Parameters Used in the Six Configurations Considered in the Analysis and Their Corresponding Collapse and Blowout Pressures

\begin{tabular}{lcccccccc}
\hline & & & & & \multicolumn{3}{c}{ Deterministic results } \\
\cline { 6 - 8 } & $\mu_{\varphi}$ & & & & & \\
Soil & (degrees) & $\mu_{c}(\mathrm{kPa})$ & $\mathrm{D} . \mu_{\gamma}\left(\mathrm{kN} / \mathrm{m}^{2}\right)$ & $\mu_{\mathrm{C}} / \mathrm{D}$ & Model & $\sigma_{c}(\mathrm{kPa})$ & $\sigma_{b}$ \\
\hline S1 & 40 & - & 150 & 1 & M1 & 12.4 & 2,984 \\
S2 & 25 & N/A & 250 & 1 & M1 & 49.0 & 2,333 \\
DC1 & 17 & 7 & 180 & 1 & M1 & 37.8 & 1,097 \\
DC2 & 20 & 20 & 250 & 1 & M1 & 13.6 & 2,056 \\
UC1 & - & 20 & 150 & 1 & M2 & 81.6 & 401.4 \\
UC2 & N/A & 30 & 250 & 1.5 & M2 & 244.1 & 810.5 \\
\hline
\end{tabular}

Table 4. Description of the Probabilistic Scenarios for the Five Random Variables

\begin{tabular}{|c|c|c|c|c|}
\hline \multirow[b]{2}{*}{ Variable } & \multicolumn{3}{|c|}{$\operatorname{COV}(\%)$} & \multirow[b]{2}{*}{$\begin{array}{c}\text { Type of } \\
\text { distribution }\end{array}$} \\
\hline & $\begin{array}{l}\text { Optimistic } \\
\text { scenario }\end{array}$ & $\begin{array}{l}\text { Neutral } \\
\text { scenario }\end{array}$ & $\begin{array}{c}\text { Pessimistic } \\
\text { scenario }\end{array}$ & \\
\hline $\begin{array}{l}\text { Internal friction } \\
\text { angle } \varphi \\
\text { (degrees) }\end{array}$ & 5 & 10 & 15 & $\begin{array}{l}\text { Gaussian or } \\
\text { Beta }\end{array}$ \\
\hline Cohesion $c(\mathrm{kPa})$ & 10 & 20 & 30 & $\begin{array}{l}\text { Gaussian or } \\
\text { Lognormal }\end{array}$ \\
\hline $\begin{array}{l}\text { Unit weight } \\
\gamma\left(\mathrm{kN} / \mathrm{m}^{3}\right)\end{array}$ & 3 & 5 & 8 & $\begin{array}{l}\text { Gaussian or } \\
\text { Lognormal }\end{array}$ \\
\hline Cover depth (m) & 1 & 3 & 5 & $\begin{array}{l}\text { Gaussian or } \\
\text { Lognormal }\end{array}$ \\
\hline $\begin{array}{l}\text { Applied face } \\
\text { pressure } \sigma_{t}(\mathrm{kPa})\end{array}$ & 5 & 15 & 25 & $\begin{array}{l}\text { Gaussian or } \\
\text { Lognormal }\end{array}$ \\
\hline
\end{tabular}

a lognormal distribution. A possible correlation may exist between $\varphi$ and $c$, although there is no large consensus in the literature on the value of this correlation. In the present article, when dealing with frictional and cohesive soils (DC1 and DC2), it is chosen to use correlation values of $-0.4,0$, and +0.4 in the optimistic, neutral, and pessimistic scenarios, respectively.

\section{Method Used for Uncertainty Propagation}

As is well known, the most robust and accurate probabilistic approach is the Monte Carlo simulation method. This method makes possible the propagation of the uncertainties from the input data to the model output through a deterministic model to compute the PDF of the system response. However, this method requires a large number of calls of the deterministic model (about 1 million samples for a target failure probability of $10^{-5}$ ). This number of calls is obviously out of reach for most deterministic models because of their computational costs. To overcome this shortcoming, a powerful probabilistic method called CSRSM is used in this paper. The principles of this method are briefly described herein. The reader may find a more detailed description in Isukapalli (1999), Sudret (2008), Phoon and Huang (2007), Huang et al. (2009), Mollon et al. (2011a), and Li et al. (2011).

The CSRSM allows one to obtain an analytical approximation (called a meta-model) of a random system response. Thus, the system response may be computed more quickly when using any classical probabilistic method (such as Monte Carlo sampling). This is because one uses the meta-model and not the original deterministic model. In the framework of CSRSM, the meta-model is a polynomial chaos expansion (PCE) of a given order $n$. In this paper, it is expressed in the basis of the multidimensional Hermite polynomials of orders $\leq n$, although other types of polynomials may also be used (cf. Xiu and Karniadakis 2002). For a given set of the probabilistic parameters of the input random variables, the unknown coefficients of the PCE are obtained hereafter by regression using the response of the original deterministic model at a given number of so-called collocation points. This number is much smaller than the number of simulations required by the crude Monte Carlo simulation methodology applied on the original deterministic model. As proposed in Mollon et al. (2011a), PCEs of order 4 are used in this study. Different number of random variables are used for the different soil configurations (see Table 3): (1) three random variables for $\mathrm{S} 1$ and $\mathrm{S} 2$ (these variables are $\varphi, \gamma$, and $C$ ); (2) three random variables for $\mathrm{UC} 1$ and $\mathrm{UC} 2(c, \gamma$, and $C)$; and (3) four random variables for $\mathrm{DC} 1$ and $\mathrm{DC} 2(\varphi, c, \gamma$, and $C)$. A very detailed description involving the application of CSRSM to the face collapse was proposed in Mollon et al. (2011a). This study was not expected to provide accurate results because it used a simple and biased deterministic model and considered a limited number of random variables. Thus, the paper by Mollon et al. (2011a) mainly had a methodological value, and the readers are expected to find in that paper the theoretical background of CSRSM to reproduce the present analysis. For this reason, the present paper does not provide significant details on the application of CSRSM to the proposed deterministic models and focuses only on the results.

Fig. 6 presents the PDFs of $\sigma_{c}$ and $\sigma_{b}$ in the case of the soil configuration DC1, for the three probabilistic scenarios and for the two types of variables (similar trends were obtained for the five other configurations). These PDFs are obtained by Monte Carlo simulations ( 1 million samples) performed on the meta-models obtained after the application of CSRSM. Fig. 6 emphasizes the important influence of the probabilistic scenario on the PDFs of the critical pressures. Indeed, an optimistic scenario leads to rather narrow distributions of the critical pressures, while a pessimistic scenario leads to much more spreadout distributions and thus to a larger variability. On the contrary, the influence of the type of variables seems rather limited. This is 


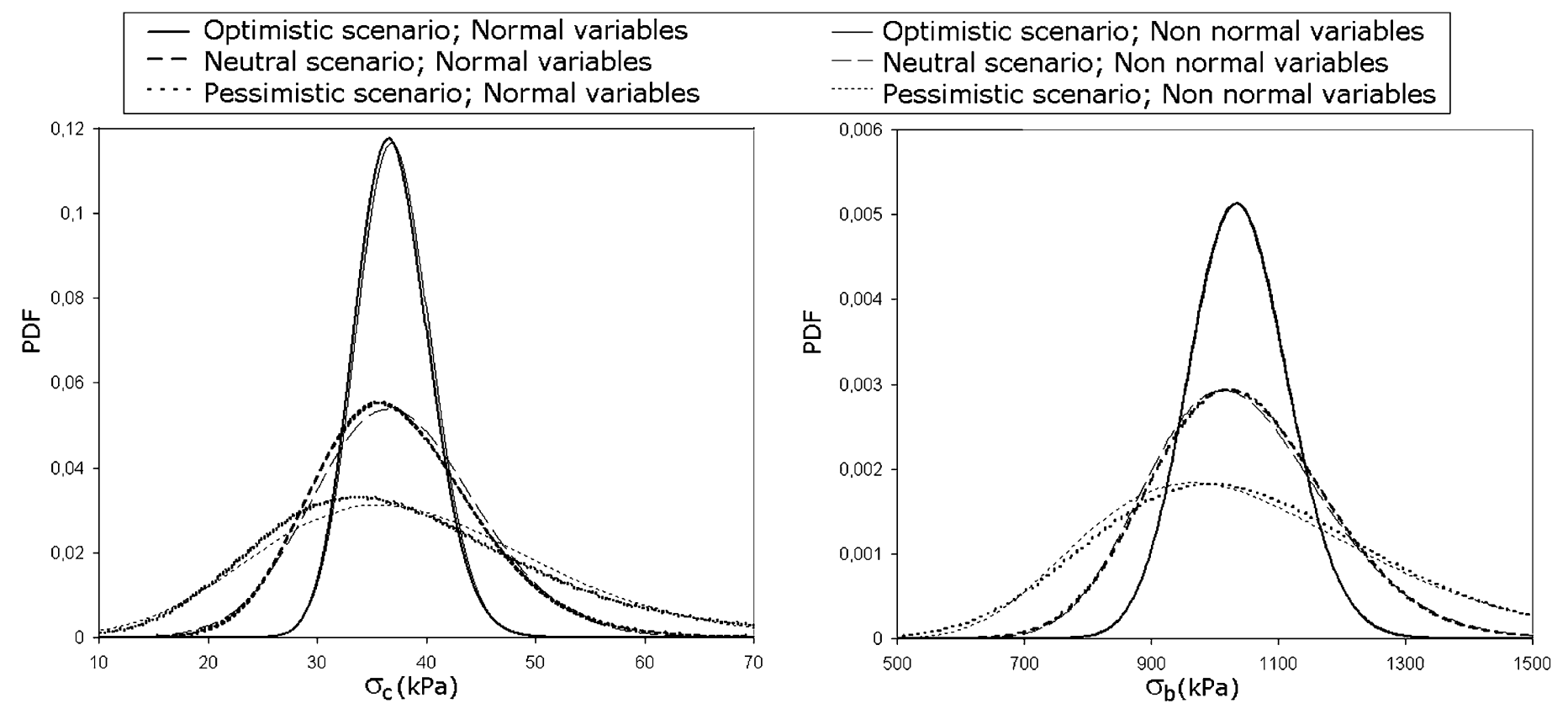

Fig. 6. Probability density functions (PDFs) of the critical collapse and blowout pressures for the soil configuration DC1

confirmed by the results presented in Table 5, which provides the COVs of $\sigma_{c}$ and $\sigma_{b}$ for the six soil configurations, for the three probabilistic scenarios, and for the two types of variables. This table shows that the COVs can have very different values, running from 7.0 to $124.4 \%$ for $\sigma_{c}$ and from 4.7 to $49.6 \%$ for $\sigma_{b}$.

\section{Failure Probability against Collapse and Blowout}

It would be desirable to assess for a chosen face pressure $\mu_{\sigma \mathrm{t}}$ the probability of failure against a single mode of failure (collapse or blowout) of the tunnel face, or more importantly the probability of failure against both collapse and blowout. The failure probability against collapse (respectively, blowout) corresponds, respectively, to the event $G_{1}<0$ (respectively, $G_{2}<0$ ) where

$$
\begin{aligned}
& G_{1}=\sigma_{t}-\sigma_{c} \\
& G_{2}=\sigma_{b}-\sigma_{t}
\end{aligned}
$$

The global failure probability against collapse and blowout corresponds to the event $\left(G_{1}<0\right.$ or $\left.G_{2}<0\right)$. For a given probabilistic scenario and for a given type of random variables (normal or nonnormal), it is possible to run Monte Carlo simulations and evaluate the probability of failure against collapse or/and blowout very quickly. A number of samples of 1 million is chosen to achieve a satisfactory level of accuracy for a target failure probability as low as $10^{-5}$. Notice that for each sample, the value of $\sigma_{t}$ is obtained directly by generating a random value from the PDF of $\sigma_{t}$, because this PDF is known and no uncertainty propagation is needed; however, the value of $\sigma_{c}$ (or/and $\sigma_{b}$ ) is obtained by generating a vector of standard random variables (whose dimension is equal to the number of the uncertain parameters) and by replacing this vector into the meta-model of $\sigma_{c}$ (and/or $\sigma_{b}$ ) to compute the value of $G_{1}$ (and/or $G_{2}$ ) using Eqs. (1) and (2) at a quasi-null time cost.

The failure probability against a single mode of failure (collapse or blowout) is provided in Figs. 7 and 8, respectively, for the sands (S1 and S2) and for the drained clays (DC1 and DC2). In each of these
Table 5. Coefficients of Variation of $\sigma_{c}$ and $\sigma_{b}$ for the Six Soil Configurations for (1) the Three Probabilistic Scenarios and (2) the Two

\begin{tabular}{|c|c|c|c|c|c|c|}
\hline \multirow[b]{2}{*}{ Case } & \multicolumn{2}{|c|}{ Optimistic scenario } & \multicolumn{2}{|c|}{ Neutral scenario } & \multicolumn{2}{|c|}{ Pessimistic scenario } \\
\hline & $\begin{array}{c}\text { Normal } \\
\text { variables }\end{array}$ & $\begin{array}{c}\text { Nonnormal } \\
\text { variables }\end{array}$ & $\begin{array}{c}\text { Normal } \\
\text { variables }\end{array}$ & $\begin{array}{c}\text { Nonnormal } \\
\text { variables }\end{array}$ & $\begin{array}{c}\text { Normal } \\
\text { variables }\end{array}$ & $\begin{array}{c}\text { Nonnormal } \\
\text { variables }\end{array}$ \\
\hline \multicolumn{7}{|l|}{$\mathrm{S} 1$} \\
\hline$\sigma_{c}$ & 11.6 & 11.8 & 23.0 & 24.1 & 34.8 & 37.8 \\
\hline$\sigma_{b}$ & 15.0 & 14.8 & 30.5 & 30.9 & 46.7 & 49.6 \\
\hline \multicolumn{7}{|l|}{ S2 } \\
\hline$\sigma_{c}$ & 7.0 & 8.5 & 15.9 & 16.6 & 24.3 & 25.3 \\
\hline$\sigma_{b}$ & 7.5 & 7.5 & 15.1 & 15.3 & 22.9 & 23.6 \\
\hline \multicolumn{7}{|l|}{ DC1 } \\
\hline$\sigma_{c}$ & 8.9 & 8.8 & 20.0 & 19.9 & 35.3 & 33.9 \\
\hline$\sigma_{b}$ & 6.4 & 6.4 & 12.6 & 12.8 & 21.3 & 21.7 \\
\hline \multicolumn{7}{|l|}{ DC2 } \\
\hline$\sigma_{c}$ & 35.4 & 34.6 & 83.6 & 82.0 & 124.4 & 116.2 \\
\hline$\sigma_{b}$ & 6.7 & 6.8 & 13.8 & 13.9 & 23.6 & 23.9 \\
\hline \multicolumn{7}{|l|}{$\mathrm{UC} 1$} \\
\hline$\sigma_{c}$ & 21.2 & 21.2 & 41.3 & 41.0 & 62.7 & 61.2 \\
\hline$\sigma_{b}$ & 4.8 & 4.7 & 9.9 & 9.8 & 15.0 & 14.6 \\
\hline \multicolumn{7}{|l|}{$\mathrm{UC} 2$} \\
\hline$\sigma_{c}$ & 13.2 & 13.2 & 25.6 & 25.4 & 39.0 & 38.1 \\
\hline$\sigma_{b}$ & 4.7 & 4.7 & 8.9 & 8.8 & 13.5 & 13.2 \\
\hline
\end{tabular}
types of PDF

figures, the curves on the left-hand side correspond to a collapse of the face, and the curves on the right-hand side correspond to a blowout. The failure probabilities are very high for low values and high values of the applied face pressure $\mu_{\sigma t}$ corresponding, respectively, to collapse and blowout of the soil. In the central part of these figures, one may observe an interval of the $\mu_{\sigma t}$ value for which both failures by collapse and blowout have probabilities of occurrence smaller than $10^{-5}$. Such an interval can therefore be considered as the range of the safe values of the applied face pressure $\mu_{\sigma t}$. The value of $10^{-5}$ was chosen here as an example, but a different acceptable probability of failure may be chosen depending on the criticality of the existing buildings at the ground surface and/or on local regulations. Figs. 7 and 


\begin{tabular}{|ll|}
\hline - Optimistic scenario; Normal variables & - Optimistic scenario; Non normal variables \\
- - Neutral scenario; Normal variables & -- Neutral scenario; Non normal variables \\
- Pessimistic scenario; Normal variables & ....... Pessimistic scenario; Non normal variables
\end{tabular}
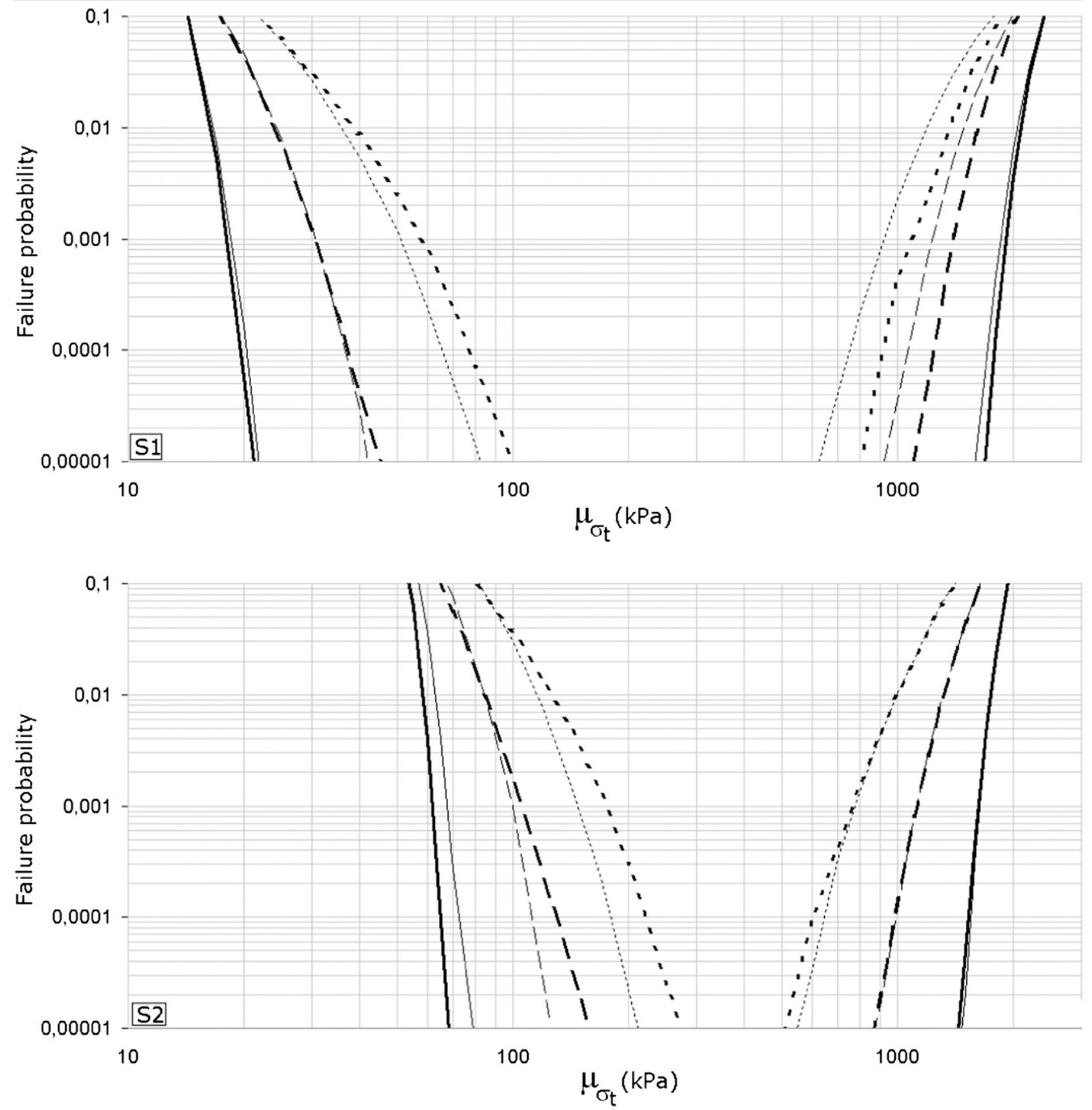

Fig. 7. Failure probability against a single mode of failure (collapse for the left-hand curves and blowout for the right-hand curves) versus the applied face pressure $\mu_{\sigma t}$ for the soil configurations S1 and S2

8 show that, for frictional soils (with or without cohesion), the width of this interval is highly dependant on the soil configuration and on the probabilistic scenario. An optimistic scenario leads to a very wide safe interval, while a pessimistic scenario strongly reduces its width. The type of variables (normal or nonnormal) may also have an influence on this interval, but no general trend appears. For the soil configuration DC1 (in the case of a pessimistic scenario and normal variables), the safe interval is rather narrow (from 250 to $350 \mathrm{kPa}$ ).

The two configurations corresponding to purely cohesive soils (UC1 and UC2) show a different behavior. Indeed, Fig. 9(a) shows an example of this behavior for the UC1 soil in the case of normal variables and a neutral scenario. It appears that the safety interval pointed out in the case of frictional soils does not exist anymore because the curves of failure probability against collapse and blowout meet before reaching a value smaller than $10^{-5}$. This is mostly related to the fact that the blowout in purely cohesive soils occurs at much smaller values of the face pressure than in frictional soils (Table 3). For this reason, it appears that there is no value of $\mu_{\sigma t}$ for which both failure probabilities would be lower than $10^{-5}$. Because the two failure modes cannot occur simultaneously, it is 

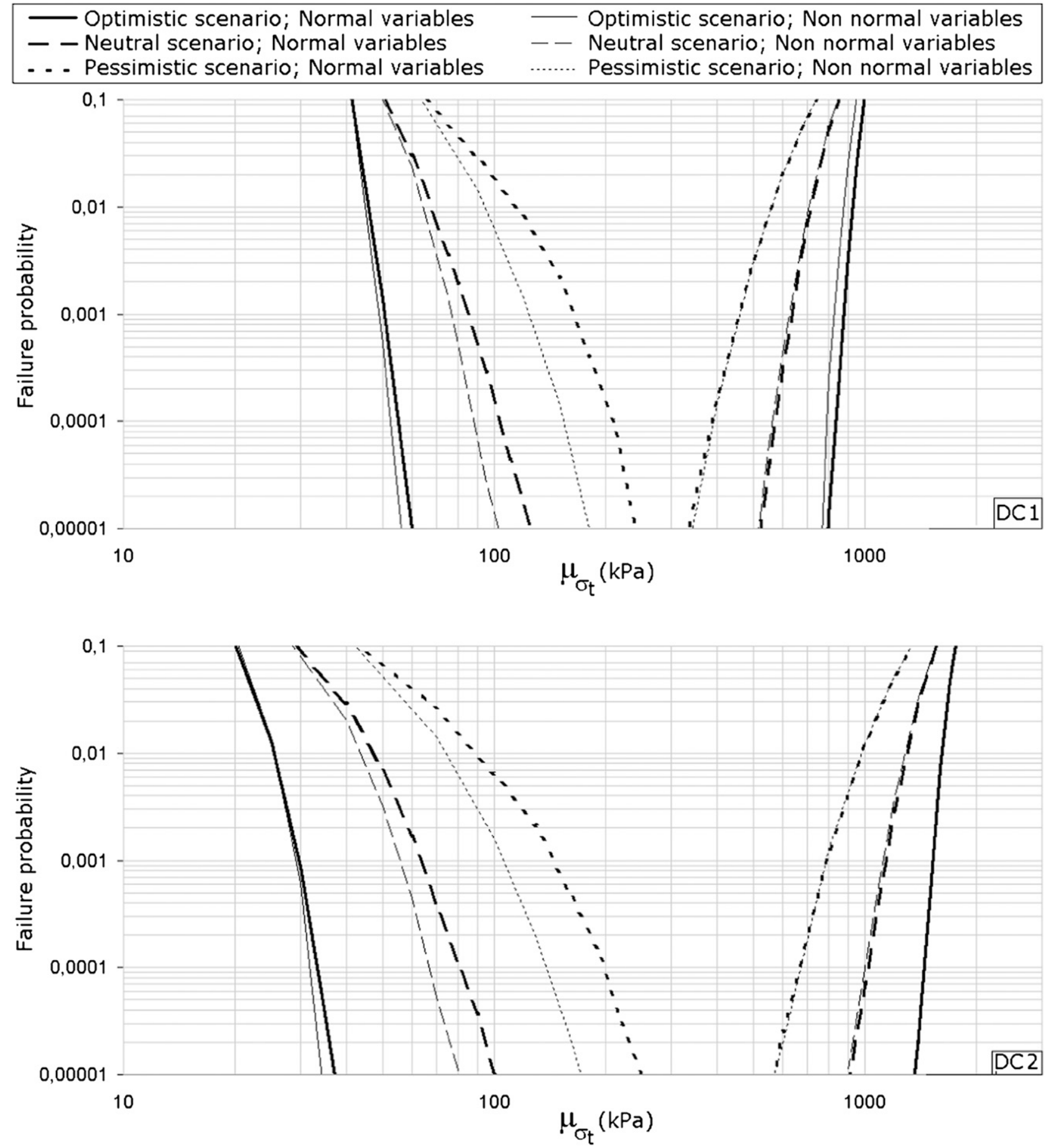

Fig. 8. Failure probability against a single mode of failure (collapse for the left-hand curves and blowout for the right-hand curves) versus the applied face pressure $\mu_{\sigma t}$ for the soil configurations DC1 and DC2

possible to define a global failure probability by adding the values of the failure probability of the two modes. This operation is performed in Fig. 9(b) and leads to a single curve of the failure probability with a minimum value. Notice that the values of the global failure probability could also be obtained by applying Monte Carlo simulation methodology on the meta-model under the condition $\left(G_{1}<0\right.$ or $G_{2}<0$ ). It should be emphasized here that the value of the applied tunnel pressure corresponding to the minimum failure probability is that for which neither modes or failure (collapse or blowout) is predominant; however, smaller and greater values of the applied tunnel pressure lead to greater values of the global failure probability because of the predominance of the collapse mode of failure for the smaller values of the applied pressure (the effect of blowout being less significant in this case) and the predominance of the blowout mode of failure for the larger values of this applied pressure (the effect of collapse being less significant in this case).

Fig. 10 presents similar curves to Fig. 9(b), but for the three probabilistic scenarios, for the two types of variables, and for the two soil configurations UC1 and UC2. Once again, it appears that the probabilistic scenario has an important influence on the failure 

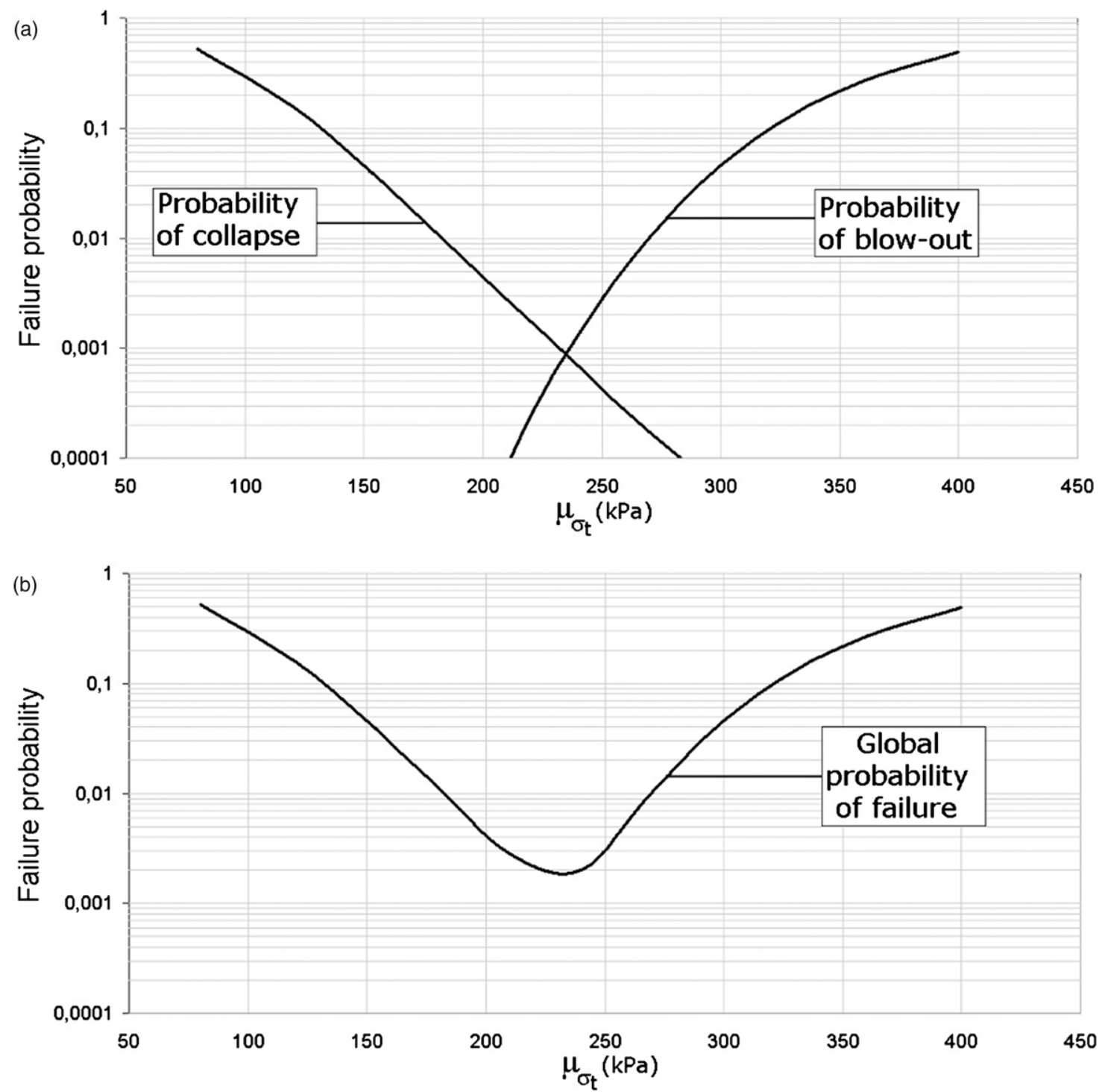

Fig. 9. (a) Failure probability against single mode of failure (collapse or blowout) versus the applied face pressure $\mu_{\sigma t}$ for the UC1 soil; (b) failure probability against both mode of failure (collapse or blowout) versus the applied face pressure $\mu_{\sigma t}$ for the same soil

probability. In the case of an optimistic scenario, one may observe a safety interval (for which the failure probability is lower than $10^{-5}$ ) similar to the one observed for frictional soils. However, in the neutral and pessimistic scenarios, it is not possible to achieve a failure probability lower than $10^{-5}$. In the case of normal variables and for the soils UC1 and UC2, respectively, the lowest failure probabilities that one may reach are equal to 0.0018 and 0.0061 in a neutral scenario and to 0.047 and 0.082 in a pessimistic scenario. These values are surprisingly high, especially when one considers that they are minimum values and that any other choice of $\mu_{\sigma t}$ can only increase them. The use of nonnormal random variables seems to reduce both the minimum value of the failure probability and the corresponding value of $\mu_{\sigma t}$, but this cannot be considered as certain based on this limited number of cases. One should also notice that the value of the minimum failure probability is not linked to the width of the deterministic range of safety (that is, the interval between the deterministic values of $\sigma_{c}$ and $\sigma_{b}$ ). Indeed, this interval is much larger for $\mathrm{UC} 2$ than for $\mathrm{UC} 1$ (see Table 3 ), while the maximum level of safety is larger for $\mathrm{UC} 1$ than for UC2.

As a first attempt to find some general trends on the safety of pressurized tunnels in purely cohesive soils, several $\mu_{\sigma t}-\mathrm{P}_{\mathrm{f}}$ curves are plotted in Fig. 11 for eight purely cohesive soils. These eight cases cover several values of the soil cohesion and unit weight and several values of the tunnel diameter and cover depth in the case of normal variables and for the neutral probabilistic scenario. Fig. 11 shows that the mean value of the cohesion $\mu_{c}$ does not modify the optimal value of $\mu_{\sigma t}$ but highly influences the corresponding value of the failure probability. The mean value of the cover depth $\left(\mu_{C / D}\right)$ seems to have an influence on the optimal value of $\mu_{\sigma t}$ (larger cover depth leading to larger optimal pressures), but a rather limited impact on the corresponding failure probability. Finally, an increase in the mean value of the soil unit weight $\left(\mu_{\gamma}\right)$ leads to higher values of the optimal prescribed pressure, but also to an increase of the corresponding failure probability. Based on these observations, it would appear that the optimum applied pressure evolves in a manner similar to the existing at-rest pressure before excavation (that is, independent from $c$, but increasing with $C / D$ and $\gamma$ ).

\section{Probabilistic Design}

The different theoretical approaches presented in this paper (the deterministic M1 and M2 models and the probabilistic method CSRSM) were gathered in a common platform to create the Tunnel 


\begin{tabular}{|c|c|}
\hline $\begin{array}{l}\text { - Optimistic scenario; Normal variables } \\
\text { - - Neutral scenario; Normal variables } \\
\text { - - - Pessimistic scenario; Normal variables }\end{array}$ & $\begin{array}{l}\text { - Optimistic scenario; Non normal variables } \\
-- \text { Neutral scenario; Non normal variables } \\
\ldots \ldots \text {... Pessimistic scenario; Non normal variables }\end{array}$ \\
\hline
\end{tabular}
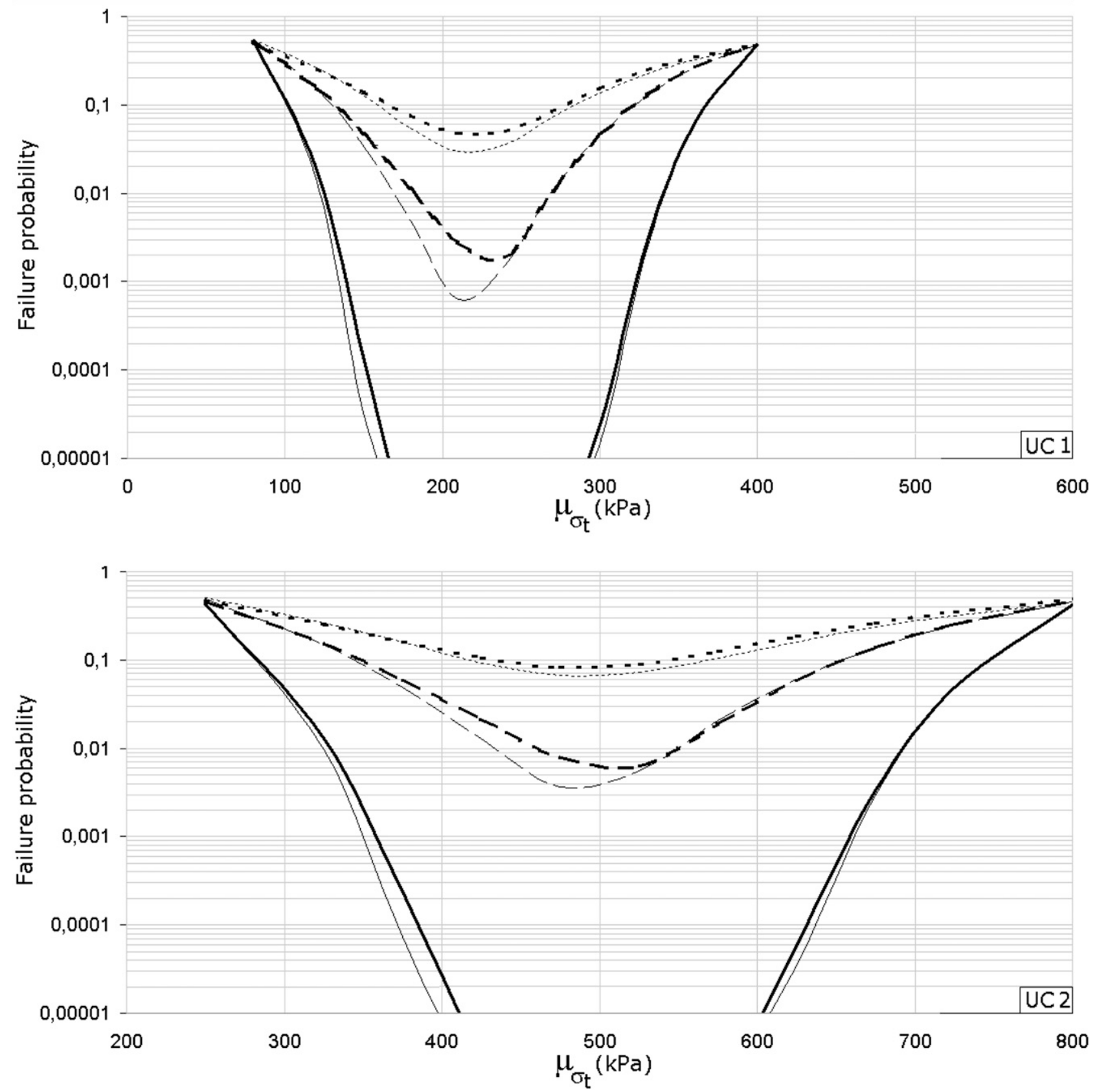

Fig. 10. Failure probability against both modes of failure (collapse and blowout) versus the applied face pressure $\mu_{\sigma t}$ for the soil configurations UC1 and UC2

Face Stability Software (TFSS) program. This software operates in a MATLAB environment, and is available for free download at the website, http://guilhem.mollon.free.fr. It is also given in the Journal website. The motivation for releasing this program is that it does not seem possible to provide a general and complete chart for the probabilistic design of the retaining face pressure. Indeed, the very large number of parameters of the problem (the tunnel geometry, the statistical characteristics of the random variables, means, COV, types of PDF, correlations between variables, and so on) prevents the plotting of such a design chart. On the other hand, it would be useful to employ this program as a preliminary design tool to identify the most influential parameters that have a significant contribution in the variability of the system response. This has the great advantage of allowing one to undertake an intensive experimental campaign on only the most influential parameters, which may lead to an economic design, as will be shown later in this section.

To demonstrate the capabilities of the TFSS program, two examples (called Example 1 and Example 2) are proposed. They are described in Table 6. Example 1 corresponds to a frictional and cohesive soil, while Example 2 corresponds to a purely cohesive soil. Illustrative probabilistic scenarios were chosen with four random variables for each example. These scenarios (mean values, COV, and type of PDF of the 


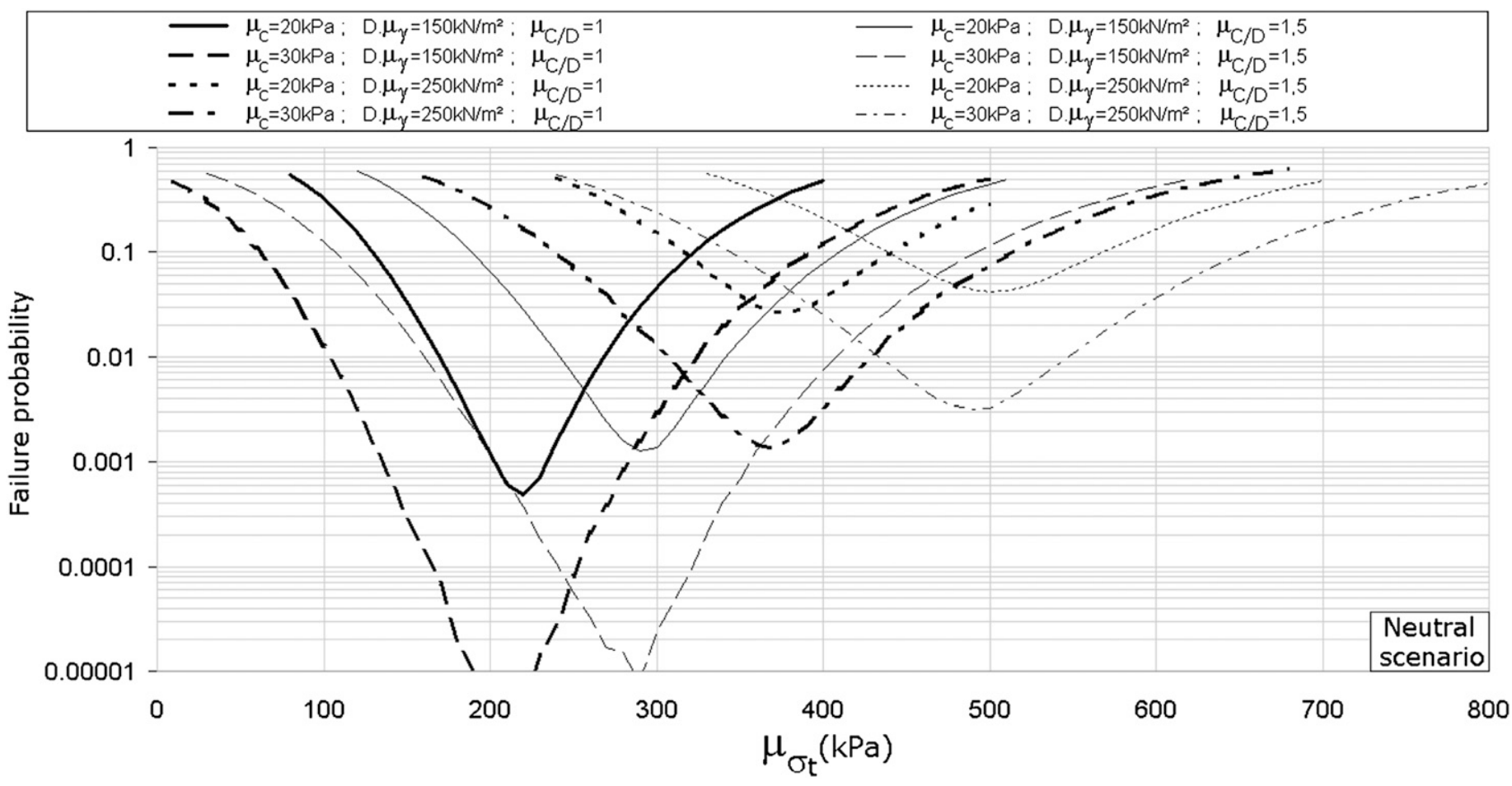

Fig. 11. Global probability of failure against both modes of failure (collapse and blowout) versus the prescribed face pressure $\mu_{\sigma t}$ for several purely cohesive soils in the case of normal variables and a neutral scenario

Table 6. Design Examples

\begin{tabular}{llll}
\hline Parameter & \multicolumn{1}{c}{ Example 1 } & \multicolumn{1}{c}{ Example 2 } & Example 2 (updated) \\
\hline $\begin{array}{l}\text { Diameter } D \\
\text { Cover depth } C\end{array}$ & $8 \mathrm{~m}$ & $11 \mathrm{~m}$ & Unchanged \\
& $12 \mathrm{~m}$ & $\begin{array}{l}\text { Mean value: } 14 \mathrm{~m} \\
\text { COV: } 5 \%\end{array}$ & Unchanged \\
& & Lognormal distribution \\
Unit weight $\gamma$ & Mean value: $20 \mathrm{kN} / \mathrm{m}^{3}$ & Mean value: $22 \mathrm{kN} / \mathrm{m}^{3}$ & COV: $5 \%$ \\
& COV: $3 \%$ & Lognormal distribution & Unchanged \\
& Lognormal distribution & $0^{\circ}$ & \\
Friction angle $\varphi$ & Mean value: $21^{\circ}$ & & Unchanged \\
& COV: $12 \%$ & Mean value: $38 \mathrm{kPa}$ & Mean value: $34 \mathrm{kPa}$ \\
Cohesion $c$ & Mean value: $5 \mathrm{kPa}$ & COV: $25 \%$ & COV: $10 \%$ \\
& COV: $18 \%$ & Lognormal distribution & Lognormal distribution \\
Correlation between $\varphi$ and $c$ & Lognormal distribution & COV: $15 \%$ & Unchanged \\
Applied face pressure $\sigma_{t}$ & -0.3 & Lognormal distribution & \\
\hline
\end{tabular}

random variables) as well as the problem geometry and some additional tuning parameters (such as the discretization parameters of the deterministic models, the order of the PCE used in CSRSM, or the number of samples used in the Monte Carlo samplings) were introduced in the TFSS program.

Because Example 1 corresponds to a tunnel excavated in a frictional soil, the failure by blowout was ignored and only collapse was considered. Indeed, preliminary computations performed by TFSS (using the M1 model) showed that the deterministic values of the collapse and blowout pressures were 27 and $1,993 \mathrm{kPa}$, indicating that the latter failure mode may be considered as unlikely because this value of applied pressure is out of reach of most pressurized shields. Some of the probabilistic outputs of the program are provided in
Fig. 12. Fig. 12(a) presents the probability density function of the critical collapse pressure based on its approximation by a PCE of order 4. Monte Carlo samplings (with $10^{6}$ samples) were then automatically performed for several values of the prescribed face pressure $\mu_{\sigma t}$ to plot the curve of Fig. 12(b), which shows the probability of collapse versus this prescribed face pressure. From this figure, it is very easy to choose a desired level of safety (such as the common value of the reliability index $\beta_{\mathrm{HL}}=3.8$ prescribed by Eurocode 7 , which corresponds to a probability of failure of $7.23 \times 10^{-5}$ ) and to determine the corresponding necessary face pressure. As shown in Fig. 12(b), this pressure should be $\mu_{\sigma t}=63 \mathrm{kPa}$ in Example 1 .

Fig. 13(a) shows similar curves for Example 2, except that both collapse and blowout failure modes were considered in the analysis 

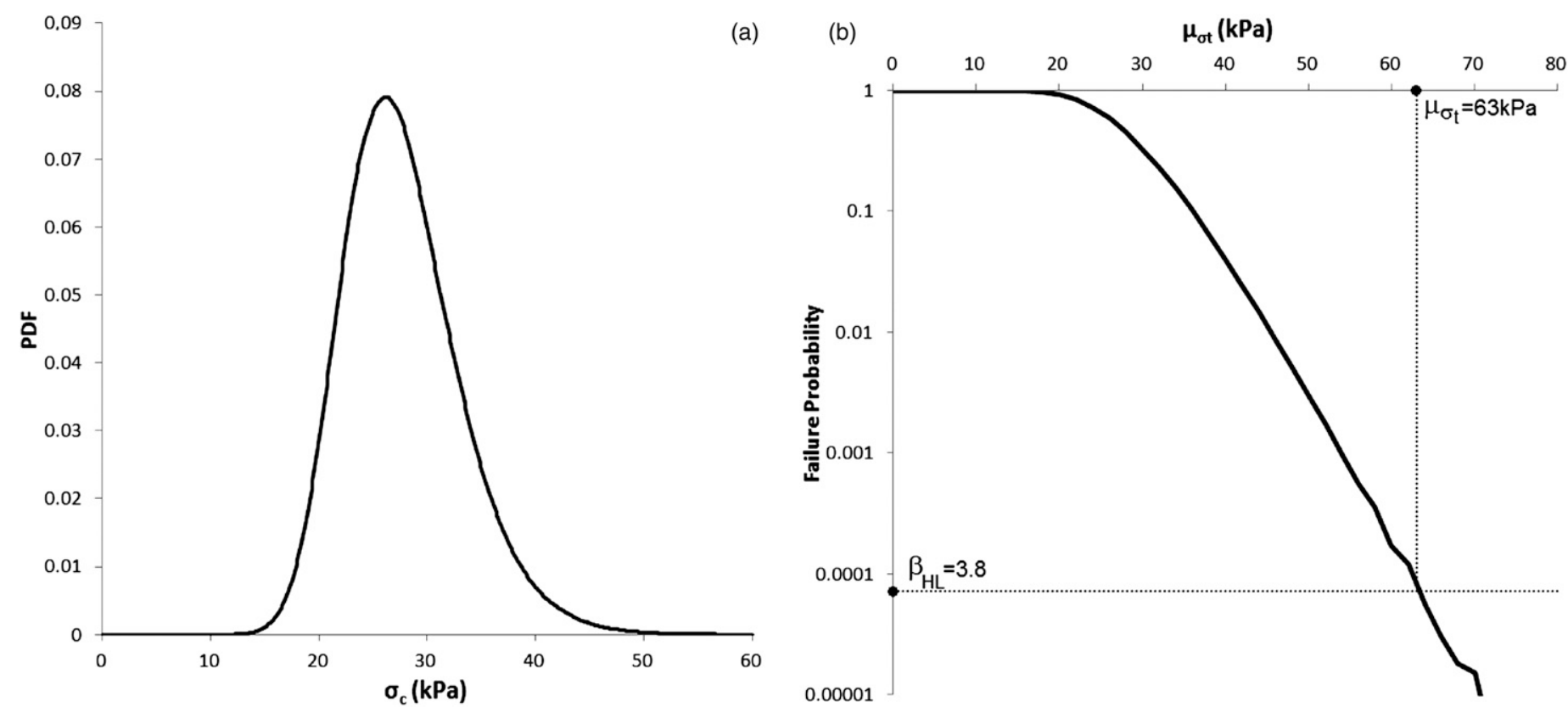

Fig. 12. Design example 1: (a) PDF of the critical collapse pressure; (b) failure probability against collapse versus the prescribed tunnel pressure

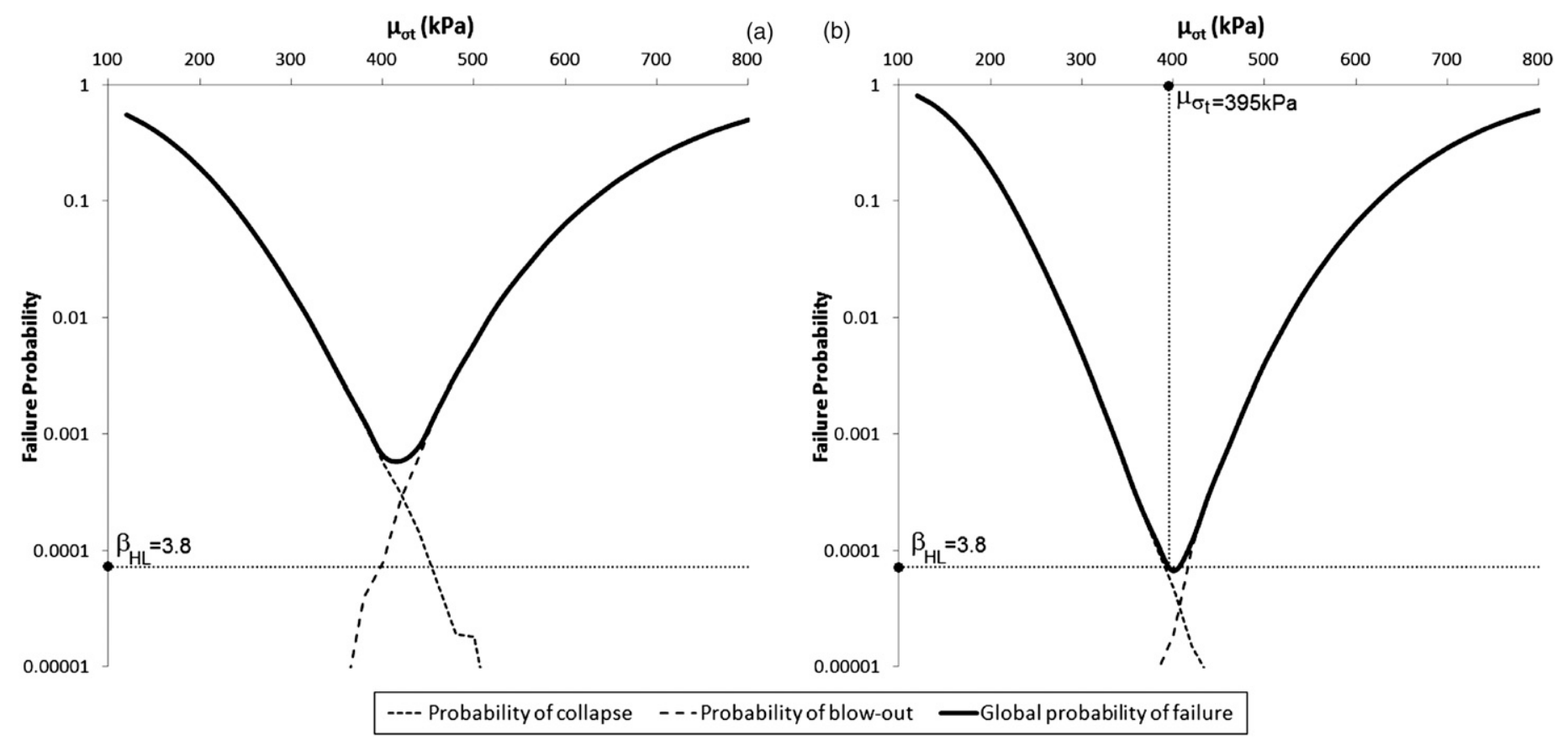

Fig. 13. Design example 2: (a) failure probability in the initial scenario; (b) failure probability in the updated scenario

because this example deals with a purely cohesive soil. This figure shows that, in the probabilistic scenario defined for Example 2, the global probability of failure (defined as the sum of the probabilities of collapse and blowout) cannot reach a satisfactory value. More specifically, there is no value of the prescribed face pressure $\mu_{\sigma t}$ for which the probability of failure is smaller than the prescribed value of $7.23 \times 10^{-5}$. If this example was a real case of excavation, the designer looking for an appropriate face pressure would thus be facing a problem that does not seem to have a solution. However, one should note that the primary reason for this observation is the high level of uncertainty of the input variables. Thus, to achieve a design with the desired level of safety, it would be necessary to gather more information only on the input random variables to decrease their level of uncertainty. The concept of the Sobol's indices was explained in detail in Mollon et al. (2011a), and is very useful for this purpose. These indices provide the weight of the different uncertain parameters in the variability of the system response (in our case, the critical collapse or blowout pressure). The TFSS program provides directly the Sobol's indices of each variable as an output, and showed that, in the present case, the variability of the cohesion was 
actually responsible for $91.8 \%$ (respectively, 90.7\%) of the variability of the critical collapse (respectively, blowout) pressure. This information is very valuable for a designer because it clearly indicates the most influential parameters that should be looked for thoroughly to reduce their level of uncertainty. The COV of the cohesion in Example 2 was $25 \%$, for a mean value of $38 \mathrm{kPa}$. Let us imagine that, based on the preceding results, the designer would chose to drive a more intensive campaign of soil recognition to increase his/her knowledge about the soil cohesion, and would obtain a corrected mean value of $34 \mathrm{kPa}$ with a much smaller COV of $10 \%$. Such updated values, when introduced in TFSS, would lead to the curves of Fig. 13(b), which are much more satisfactory. Indeed, despite the fact that the mean value of the cohesion has been found smaller than its first estimate, the reduction of its COV (that is, the improvement of the designer's knowledge) leads to a much lower value of the failure probability, and makes it possible to reach the desired level of safety of $\beta_{\mathrm{HL}}=3.8$. As shown in Fig. 13(b), a proper design value for this updated scenario would be $\mu_{\sigma t}=395 \mathrm{kPa}$. This example reveals to which extent the probabilistic methods may be used to improve the safety of the pressurized tunnels with a limited economic cost.

\section{Conclusion}

This article presents a probabilistic analysis for the determination of the admissible range of the retaining pressure that can be applied to a pressurized tunnel face without it to collapse or blowout. Two deterministic models developed by the authors in previous studies were considered for frictional and nonfrictional soils. Each model is able to provide the critical collapse pressure $\sigma_{c}$ and the critical blowout pressure $\sigma_{b}$ and, thus, the range of the admissible pressures with a satisfying accuracy and a limited time cost when compared with the classical numerical models.

Three illustrative probabilistic scenarios were chosen to model the uncertainty of the soil parameters and that of the excavation process. More precisely, these scenarios were used to account for the amount of information that is available to the designer and that is related to (1) the quality and the quantity of soil recognition and (2) the excavation monitoring (although a quantification of this relation is a broad topic that is not tackled in the current study). An efficient probabilistic method called CSRSM was used to assess the uncertainty propagation through the deterministic models. Six illustrative configurations that cover a wide range of situations were studied using this method. CSRSM makes it possible (for a given soil configuration and a given probabilistic scenario) to compute efficiently and accurately the probability of collapse and blowout of the tunnel face. Also, a probabilistic design is possible using this method. For this purpose, a MATLAB software called TFSS that makes use of CSRSM and the different deterministic models was provided and illustrated in this paper for practical use in geotechnical engineering. The main findings of this paper can be summarized as follows:

- The probabilistic scenario has a very large influence on the COV of the critical pressures: an optimistic scenario leads to a small variability of these pressures, while a pessimistic scenario increases by a lot their dispersion. It is thus desirable in a practical context to tend as much as possible toward an optimistic scenario - that is, to perform the necessary soil recognitions and excavation monitoring aiming at reducing the level of uncertainty. The computer program provided in this paper should help in identifying the most influential parameters that need further experimental investigation to lead to a safe design with a reduced economic cost.
- The failure probability is very high for low values and high values of the applied face pressure $\mu_{\sigma t}$ corresponding, respectively, to collapse and blowout. This probability is smaller for intermediate values of the applied pressure. For these smaller values of the failure probability, one can define a zone of desirable applied pressures corresponding to a target failure probability.

- In the case of frictional soils (with or without cohesion), there is an interval for $\mu_{\sigma t}$ for which the failure probability against collapse and the failure probability against blowout are smaller than $10^{-5}$. This range can be considered as a safe interval. An optimistic scenario leads to a very wide safety interval, while a pessimistic scenario reduces the width of this interval. It appears that the failure by blowout is very unlikely in frictional soils and that the design may be performed solely with respect to collapse.

- In nonfrictional soils, the safety interval pointed out in the case of frictional soils no longer exists, and there is no value of the prescribed pressure $\mu_{\sigma t}$ for which the failure probability against collapse and the failure probability against blowout are smaller than $10^{-5}$. Thus, a proper design of the face-retaining pressure in such soils requires considering both modes, and for some probabilistic scenarios it may not be possible to reach the desired level of safety. An extensive experimental campaign may thus be necessary on the most influential parameters to lead to a safe and economic design.

The main drawback of probabilistic methods is that the statistical distributions of the soil parameters (and more generally of all the uncertain parameters involved in an excavation) are never known with a good precision. Thus, it is very difficult for a designer to assess in which probabilistic scenario the design should be performed. It would be desirable in future works to build up tools to determine the level of uncertainty related to the soil properties in different contexts, in relation with the quantity and quality of the soil recognitions. If such a tool is developed in the future, the current study provides a rigorous methodology that will make it possible to perform accurate and reliable designs of the face-retaining pressure of a pressurized tunnel.

\section{Supplemental Data}

The fully working TFSS code (running in a MATLAB environment) and a text file containing a short user guide are available online in the ASCE Library (www.ascelibrary.org).

\section{References}

Anagnostou, G., and Kovari, K. (1996). "Face stability conditions with earth-pressure-balanced shields.” Tunn. Undergr. Space Technol., 11(2), $165-173$.

Augarde, C. E., Lyamin, A. V., and Sloan, S. W. (2003). "Stability of an undrained plane strain heading revisited." Comput. Geotech., 30(5), 419-430.

Broms, B. B., and Bennermark, H. (1967). "Stability of clay at vertical openings." Soil Mech. Found. Eng., 193(SM1), 71-94.

Chambon, P., and Corté, J. F. (1994). "Shallow tunnels in cohesionless soil: Stability of tunnel face." J. Geotech. Engrg., 120(7), 1148-1165.

Davis, E. H., Gunn, M. J., Mair, R. J., and Seneviratne, H. N. (1980). "The stability of shallow tunnels and underground openings in cohesive material." Geotechnique, 30(4), 397-416.

Ellstein, A. R. (1986). "Heading failure of lined tunnels in soft soils." Tunnels and Tunnelling, 18, 51-54.

FLAC3D [Computer software]. Minneapolis, ITASCA Consulting Group.

Huang, S. P., Liang, B., and Phoon, K. K. (2009). "Geotechnical probabilistic analysis by collocation-based stochastic response surface method: An 
Excel add-in implementation." Georisk: Assessment and Management of Risk for Engineered Systems and Geohazards, (3)2, 75-86.

Isukapalli, S. S. (1999). "An uncertainty analysis of transport-transformation models." Ph.D. thesis, State Univ. of New Jersey, New Brunswick, NJ.

Klar, A., Osman, A. S., and Bolton, M. (2007). "2D and 3D upper bound solutions for tunnel excavation using 'elastic' flow fields." Int. J. Numer. Anal. Methods Geomech., 31(12), 1367-1374.

Leca, E., and Dormieux, L. (1990). "Upper and lower bound solutions for the face stability of shallow circular tunnels in frictional material." Geotechnique, 40(4), 581-606.

Li, D., Chen, Y., Lu, W., and Zhou, C. (2011). "Stochastic response surface method for reliability analysis of rock slopes involving correlated nonnormal variables." Comput. Geotech., 38(1), 58-68.

MATLAB 7.0.0.19920 (R14) [Computer software]. Natick, MA, MathWorks.

Mollon, G. (2010). "Etude déterministe et probabiliste du comportement des tunnels." Ph.D. thesis, Institut National des Sciences Appliquées Lyon, Univ. of Lyon, Lyon, France (in French).

Mollon, G., Dias, D., and Soubra, A.-H. (2009a). "Probabilistic analysis and design of circular tunnels against face stability." Int. J. Geomech., 9(6), 237-249.

Mollon, G., Dias, D., and Soubra, A.-H. (2009b). "Probabilistic analysis of circular tunnels in homogeneous soils using response surface methodology." J. Geotech. Geoenviron. Eng., 135(9), 1314-1325.

Mollon, G., Dias, D., and Soubra, A.-H. (2010). "Face stability analysis of circular tunnels driven by a pressurized shield." J. Geotech. Geoenviron. Eng., (136)1, 215-229.

Mollon, G., Dias, D., and Soubra, A.-H. (2011a). "Probabilistic analysis of pressurized tunnels against face stability using collocation-based stochastic response surface method." J. Geotech. Geoenviron. Eng., 137(4), 385-397.

Mollon, G., Dias, D., and Soubra, A.-H. (2011b). "Rotational failure mechanisms for the face stability analysis of tunnels driven by pressurized shields." Int. J. Numer. Anal. Methods Geomech., 35(12), 1363-1388.

Mollon, G., Dias, D., and Soubra, A.-H. (2012). "Continuous velocity fields for collapse and blowout of a pressurized tunnel face in purely cohesive soil." Int. J. Numer. Anal. Methods Geomech., 37(13), 2061-2083

Mollon, G., Phoon, K.-K., Dias, D., and Soubra, A.-H. (2011c). "Validation of a new 2D failure mechanism for the stability analysis of a pressurized tunnel face in a spatially varying sand." J. Eng. Mech., 137(1), 8-21.

Osman, A. S., Mair, R. J., and Bolton, M. D. (2006). "On the kinematics of 2D tunnel collapse in undrained clay." Geotechnique, 56(9), 585-595.

Phoon, K. K., and Huang, H. W. (2007). "Uncertainty quantification using multi-dimensional Hermite polynomials." Probabilistic Applications in Geotechnical Engineering, Geotechnical Special Publications, ASCE, Reston, VA, 1-10.

Phoon, K.-K., and Kulhawy, F. H. (1999). "Evaluation of geotechnical property variability." Can. Geotech. J., 36(4), 625-639.

Sudret, B. (2008). "Global sensitivity analysis using polynomial chaos expansions.” Reliab. Eng. Syst. Saf., 93(7), 964-979.

Takano, D., Otani, J., Nagatani, H., and Mukunoki, T. (2006). “Application of X-ray CT boundary value problems in geotechnical engineeringResearch on tunnel face failure." Geocongress 2006, ASCE, Reston, VA, 1-6.

Xiu, D., and Karniadakis, G. E. (2002). "The Wiener-Askey polynomial chaos for stochastic differential equations." J. Sci. Comput., 24(2), 619-644. 